\title{
Characteristic profiles of DNA epigenetic modifications in colon cancer and its predisposing conditions-benign adenomas and inflammatory bowel disease
}

$\operatorname{Tomasz~Dziaman~}^{1,7^{*}}$ D, Daniel Gackowski ${ }^{1}$, Jolanta Guz ${ }^{1}$, Kinga Linowiecka', Magdalena Bodnar ${ }^{2,6}$, Marta Starczak ${ }^{1}$, Ewelina Zarakowska', Martyna Modrzejewska', Anna Szpila', Justyna Szpotan', Maciej Gawronski', Anna Labejszo ', Ariel Liebert ${ }^{4}$, Zbigniew Banaszkiewicz ${ }^{3}$, Maria Klopocka ${ }^{4}$, Marek Foksinski ${ }^{1}$, Andrzej Marszalek ${ }^{2,5}$ and Ryszard Olinski ${ }^{1,7^{*}}$

\begin{abstract}
Background: Active demethylation of 5-methyl-2'-deoxycytidine (5-mdC) in DNA occurs by oxidation to 5(hydroxymethyl)-2'-deoxycytidine (5-hmdC) and further oxidation to 5-formyl-2'-deoxycytidine (5-fdC) and 5carboxy-2'-deoxycytidine (5-cadC), and is carried out by enzymes of the ten-eleven translocation family (TETs $1,2,3)$. Decreased level of epigenetic DNA modifications in cancer tissue may be a consequence of reduced activity/expression of TET proteins. To determine the role of epigenetic DNA modifications in colon cancer development, we analyzed their levels in normal colon and various colonic pathologies. Moreover, we determined the expressions of TETs at mRNA and protein level.

The study included material from patients with inflammatory bowel disease (IBD), benign polyps (AD), and colorectal cancer (CRC). The levels of epigenetic DNA modifications and 8-oxo-7,8-dihydro-2'-deoxyguanosine (8-oxodG) in examined tissues were determined by means of isotope-dilution automated online two-dimensional ultraperformance liquid chromatography with tandem mass spectrometry (2D-UPLC-MS/MS). The expressions of TET mRNA were measured with RT-qPCR, and the expressions of TET proteins were determined immunohistochemically.

Results: IBD was characterized by the highest level of 8-oxodG among all analyzed tissues, as well as by a decrease in 5-hmdC and 5-mdC levels (at a midrange between normal colon and CRC). AD had the lowest levels of 5-hmdC and 5-mdC of all examined tissues and showed an increase in 8-oxodG and 5-(hydroxymethyl)-2'deoxyuridine (5-hmdU) levels. CRC was characterized by lower levels of 5-hmdC and 5-mdC, the lowest level of 5-fdC among all analyzed tissues, and relatively high content of 5-cadC. The expression of TET1 mRNA in CRC and AD was significantly weaker than in IBD and normal colon. Furthermore, CRC and AD showed significantly lower levels of TET2 and AID mRNA than normal colonic tissue.

(Continued on next page)
\end{abstract}

\footnotetext{
* Correspondence: tomekd@cm.umk.pl; ryszardo@cm.umk.pl

${ }^{1}$ Department of Clinical Biochemistry, Faculty of Pharmacy, Collegium

Medicum in Bydgoszcz, Nicolaus Copernicus University in Torun, Torun,

Poland

Full list of author information is available at the end of the article
}

(c) The Author(s). 2018 Open Access This article is distributed under the terms of the Creative Commons Attribution 4.0 International License (http://creativecommons.org/licenses/by/4.0/), which permits unrestricted use, distribution, and reproduction in any medium, provided you give appropriate credit to the original author(s) and the source, provide a link to the Creative Commons license, and indicate if changes were made. The Creative Commons Public Domain Dedication waiver (http://creativecommons.org/publicdomain/zero/1.0/) applies to the data made available in this article, unless otherwise stated. 
(Continued from previous page)

Conclusions: Our findings suggest that a complex relationship between aberrant pattern of DNA epigenetic modification and cancer development does not depend solely on the transcriptional status of TET proteins, but also on the characteristics of premalignant/malignant cells. This study showed for the first time that the examined colonic pathologies had their unique epigenetic marks, distinguishing them from each other, as well as from normal colonic tissue. A decrease in 5-fdC level may be a characteristic feature of largely undifferentiated cancer cells.

Keywords: DNA epigenetic modification, Ten-eleven translocation protein, Colon cancer, Inflammatory bowel disease, Adenoma, Demethylation,

\section{Background}

During the last decade, one of the hot topics in oncogenesis was the so-called cancer epigenome, having implications for cancer promotion and progression. This, in turn, is linked with a plethora of abnormalities based on somatic heritable modifications that are not caused by alterations in primary sequence of DNA.

Methylation of cytosine, usually in CpG dinucleotides, is a key epigenetic modification exerting a profound impact on gene repression, cellular identity, and organismal fate [1]. However, equally important is an opposite reaction, DNA demethylation, resulting in activation of previously silenced genes. Although a large body of evidence suggests that active demethylation may occur in mammalian cells, its molecular background is still unclear (for review, see [2]). The most plausible mechanism behind the active demethylation of 5-methyl-2' -deoxycytidine (5-mdC) moiety in DNA involves ten-eleven translocation (TET) proteins, which catalyze oxidation of 5-mdC to form 5-(hydroxymethyl)-2'-deoxycytidine (5-hmdC) and further oxidation reactions that generate 5 -formyl-2'-deoxycytidine $(5$-fdC) and 5-carboxy-2'-deoxycytidine (5-cadC) [2, 3]. Evidence from experimental studies supports the hypothesis that TET enzymes may be also involved in the synthesis of 5-(hydroxymethyl)-2'-deoxyuridine (5-hmdU), a molecule with epigenetic function [4].

Several recent studies showed that the level of 5 -hmdC in many various types of human malignancies, including $\mathrm{CRC}$, is profoundly reduced [5-7] and the degree of the reduction is proportional to tumor stage [8].

Either the mechanism or the reason behind the decrease in 5-hmdC level in cancer tissues is still not fully understood. Perhaps, this phenomenon reflects a decrease in the activity/expression of TET proteins [9]. However, it also cannot be excluded that the regulatory mechanisms of active DNA demethylation are determined by external conditions (e.g., chronic inflammation, oxidative stress, nutritional status), which results in a release of different products.

Chronic inflammation being a direct consequence of inflammatory bowel disease (IBD) is considered the most important etiological factor of sporadic colorectal malignancies [10]. Epigenetic modification of DNA is a dynamic molecular process, being a form of response to inflammation-related environmental/metabolic changes.

The downstream steps of active demethylation process may be, at least partially, responsible for the loss of 5 -mdC. Furthermore, 5 -fdC and 5-cadC were shown to be recognized by a larger number of proteins than 5 -hmdC, despite markedly higher level of the latter [11, 12].

In the vast majority of previous studies, 5-hmdC, 5-fdC, and 5-cadC were determined semi-quantitatively, by means of immunohistochemistry; consequently, the results of these might be biased due to ultra-low content of these modifications in genomic DNA of the tumor [7]. It should be also stressed that the accuracy of immunohistochemical studies depends largely on the sensitivity/specificity of antibodies against a given modification.

In our present study, instead of using a semiquantitive method with anti-5-hmdC antibodies, we determined 5-mdC, 5-hmdC, 5-fdC, 5-cadC, and 5-hmU with a highly specific and highly sensitive method developed recently in our laboratory: isotope-dilution automated online two-dimensional ultra-performance liquid chromatography with tandem mass spectrometry (2D-UPLC-MS/MS) [13].

To provide a better insight in the relationship between epigenetic DNA modifications and factors which may influence formation thereof and to determine their role in CRC development, we analyzed their levels in normal colon and various colonic pathologies, which predispose to CRC development. Moreover, we determined the expressions of TETs and AID at mRNA and protein level.

The study included samples from patients with CRC ( $n=97$, both from the tumor and from normal colonic tissue), colon adenomas (AD, $n=39)$, and IBD $(n=49)$. Since both CRC and chronic inflammation are associated with oxidative stress, aside from the epigenetic DNA modifications, we also determined an established marker of oxidatively modified DNA, 8-oxodG. The rationale of the study was to fill the gap in existing knowledge, explaining how conditions which predispose to CRC development can influence the synthesis of TET-mediated DNA modifications and oxidatively modified DNA. 


\section{Methods}

\section{Study group}

The study material originated from three groups of patients with (1) IBD ( $n=49$, median age 35 years, $53 \%$ of women), (2) AD, i.e., histologically confirmed adenoma tubulare $(90 \%)$ or adenoma tubulovillosum $(10 \%)(n=39$, median age 65 years, $46 \%$ of women), and (3) CRC, i.e., histologically confirmed stage A (8\%), stage B (45\%), stage C (29\%), or stage D (9\%) adenocarcinoma, or malignant polyps (9\%) ( $n=97$, median age 65 years, $46 \%$ of women). None of the study subjects were related with one another, and all of them were Caucasians. All participants of the study were recruited in a hospital setting (Collegium Medicum, Nicolaus Copernicus University, Bydgoszcz, Poland) and subjected to colonoscopy. At the enrollment, all subjects completed a questionnaire containing information about their demographics, smoking, diet, and medical history. The study groups were matched for eating habits, age, body weight, and smoking status. No significant intergroup differences were found in terms of body weight and body stature of male and female subjects. To make the study groups even more homogenous, the subjects who reported overeating or use of dietary supplements during a month preceding the study were not included in the analysis. The questionnaire survey was conducted by the team physician (Dr. Banaszkiewicz, Dr. Klopocka).

\section{Preparation of tissue microarrays (TMA) for immunochemical analysis}

Immunohistochemical studies were performed using archived formaldehyde-fixed paraffin-embedded (FFPE) tissue sections derived in the Department of Clinical Pathomorphology, Collegium Medicum in Bydgoszcz, Nicolaus Copernicus University in Torun.

Hematoxylin and eosin (H\&E)-stained microscopic slides of archived FFPE tissue sections (donor blocks) were used to identify representative tumor areas with at least $80 \%$ tumor cells. Then, two such regions, each $2 \mathrm{~mm}$ in diameter, were transferred from the donor blocks to a recipient TMA block using an automated tissue arrayer (TMA Master3D HISTECH, Budapest, Hungary). The same procedure was repeated for normal tissue located at least $2 \mathrm{~cm}$ from the tumor resection margin. Then, another set of H\&E-stained slides was prepared to verify the accuracy of the TMA blocks. TMA blocks were verified and double-checked by two independent pathologists.

\section{Immunohistochemistry}

Immunohistochemical staining was carried out as described elsewhere [14-16], and the results were standardized against a series of positive and negative controls. Positive control staining was performed on a model tissue section selected according to The Human Protein Atlas (http://www.proteinatlas.org) [17] and antibody specification (Additional file 1: Table S1) and the antibody datasheet. Negative controls were prepared from the examined tissues treated with $1 \%$ solution of bovine serum albumin (BSA) in phosphate buffered saline (PBS), instead of the primary antibody. Paraffin TMA blocks and archived FFPE tissue sections were cut with a manual rotary microtome (AccuCut, Sakura, Torrance, USA) to obtain 4- $\mu \mathrm{m}$ slices, which were then processed routinely and mounted on extra adhesive slides (SuperFrostPlus, MenzelGlasser, Braunschweig, Germany).

The deparaffinization, rehydration, and antigen retrieval were carried out in PT-Link system (Dako, Agilent Technologies, USA). The slides were heated for $20 \mathrm{~min}$ in Epitope Retrieval Solution high-pH $\left(95-98{ }^{\circ} \mathrm{C}\right.$; Dako, Agilent Technologies). Then, the activity of endogenous peroxidase was blocked by a 15 -min incubation with $3 \%$ $\mathrm{H}_{2} \mathrm{O}_{2}$ solution, and non-specific binding was eliminated by a 15-min incubation with 5\% BSA solution; both reactions were carried out at room temperature. Subsequently, the slides were incubated with primary antibodies against TET1, TET2, and TET3 (specified in Additional file 1: Table S1). The antibody complexes were detected with EnVision Flex Anti-Mouse/Rabbit HRP-Labeled Polymer (Dako, Agilent Technologies) and localized using 3-3'diaminobenzidine (DAB) as a chromogen. Finally, the slides were counterstained with hematoxylin, subsequently dehydrated, cleared in series of xylenes, and coverslipped using mounting medium (Dako, Agilent Technologies).

\section{Evaluation of protein expression based on immunohistochemical staining}

Each slide was examined under ECLIPSE E400 light microscope (Nikon Instruments Europe, Amsterdam, Netherlands) with the low-power (20x) objective. The result of immunohistochemical staining was expressed according to Immunoreactive Remmele-Stegner (IRS) score [18], described in detail in our previous papers [14-16, 19]. Total IRC score (from 0 to 12) was obtained by multiplying the staining intensity score $(0-$ negative, 1 -weak staining, 2-moderate staining, 3-strong staining) by the relative proportion of immunolabeled specimen area $(0-$ none; $1-$ less than $10 \% ; 2-10$ to $50 \%$; $3-$ 50 to $80 \%$; 4 -at least $80 \%)$.

\section{Extraction of DNA from tissues and its hydrolysis to deoxynucleosides}

DNA from examined fresh frozen tissues was isolated as described elsewhere [20], with some modifications. Isolated DNA was dissolved in $100 \mathrm{mM}$ ammonium acetate (Sigma-Aldrich) containing $0.1 \mathrm{mM} \mathrm{ZnCl} 2$ (pH 4.3). The dissolved DNA samples $(50 \mu \mathrm{l})$ were mixed with $1 \mathrm{U}$ of 
nuclease P1 (Sigma-Aldrich) and tetrahydrouridine (Calbiochem) (as cytidine deaminase inhibitor, $10 \mu \mathrm{g}$ per sample) and incubated at $37{ }^{\circ} \mathrm{C}$ for $1 \mathrm{~h}$. Subsequently, $12 \mu \mathrm{l} 5 \%(v / v) \mathrm{NH}_{4} \mathrm{OH}$ (JT Baker) and $1.3 \mathrm{U}$ of alkaline phosphatase (Sigma-Aldrich) were added to each sample following 1-h incubation at $37{ }^{\circ} \mathrm{C}$. Finally, all DNA hydrolysates were acidified with $\mathrm{CH}_{3} \mathrm{COOH}$ (Sigma-Aldrich) (to final $v / v$ concentration of $2 \%$ ) and ultrafiltered prior to injection.

\section{Isolation of DNA and determination of epigenetic modifications and 8-oxodG in DNA isolates}

The methodology used to determine 5-methyl-2' -deoxycytidine (5-mdC), 5-hydroxymethyl-2'-deoxycytidine (5-hmdC), 5-formyl-2'-deoxycytidine (5-fdC), 5-carboxy -2'deoxycytidine (5-cadC), 5-(hydroxymethyl)-2'-deoxyuridine $(5-\mathrm{hmdU})$, and 8-oxodG levels by means of 2D-UPLC-MS/MS has been described elsewhere [13]. Transition patterns and specific detector settings for all analyzed compounds are presented in the Additional file 2 : Table S2.

\section{Gene expression analysis}

Isolated leukocytes were stored at $-80{ }^{\circ} \mathrm{C}$ until the analysis. RNA was isolated with MagNA Pure 2.0 (Roche) following the standard procedures. Concentration and purity of RNA aliquots were verified spectrophotometrically with NanoDrop 2000 (Thermo Scientific). A $A_{260}$ / $\mathrm{A}_{280}$ ratio was used as an indicator of protein contamination and $\mathrm{A}_{260} / \mathrm{A}_{230}$ ratio as a measure of contamination with polysaccharides, phenol, and/or chaotropic salts. Quality and integrity of total RNA were assessed by visualization of $28 \mathrm{~S} / 18 \mathrm{~S} / 5.8 \mathrm{~S}$ rRNA band pattern in a $1.2 \%$ agarose gel. Non-denaturing electrophoresis was carried out at $95 \mathrm{~V}$ for $20 \mathrm{~min}$ in TBE buffer (Tris Boric Acid - EDTA). The gel was stained with ethidium bromide or SimplySafe and visualized using GBox EF Gel Documentation System (SynGene). Purified RNA was stored at $-80^{\circ} \mathrm{C}$. The samples with RNA concentrations greater than $50 \mathrm{ng} / \mu \mathrm{l}$ were qualified for further analysis. 0.5 microgram of total RNA from each sample (in 20- $\mu$ l volume) was used for cDNA synthesis by reverse transcription with High-Capacity cDNA Reverse Transcription Kit (Applied Biosystems, catalog no. 43-688-14), according to the manufacturer's instruction. The reaction was carried out with Mastercycler Nexus Gradient thermocycler (Eppendorf). To exclude contamination with genomic DNA, reverse transcriptase reaction included also a negative control. cDNA was either used for qPCR setup immediately after obtaining or stored at $-20{ }^{\circ} \mathrm{C}$. The RT-qPCR complies with the Minimum Information for Publication of Quantitative Real-time PCR Experiments (MIQE) guidelines. Three gene transcripts, TET1, TET2, and TET3, were analyzed by relative quantitative RT-PCR (RT-qPCR) with relevant primers and short hydrolysis probes substituted with Locked Nucleic Acids from the Universal Probe Library (UPL, Roche) (see: Additional file 3: Table S3). The probes were labeled with fluorescein (FAM) at the 5 '-end and with a dark quencher dye at the 3 '-end. Expressions of target genes were normalized for two selected reference genes, $H M B S$ (GeneID: 3145) and TBP (GeneID: 6908), using UPL Ready Assay $\# 100092149$ and $\# 100092158$, respectively. Real-time PCR mixes (in $20 \mu \mathrm{l}$ volumes) were prepared from cDNA following the standard procedures for LightCycler480 Probes Master (Roche), provided with the reagent set. The reactions were carried out on 96-well plates. Aside from the proper samples, each plate included also no-template control and no-RT control. Quantitative real-time PCR was carried out with LightCycler 480 II, using the following cycling parameters: $10 \mathrm{~s}$ at $95^{\circ} \mathrm{C}$, followed by 45 repeats $10 \mathrm{~s}$ each at $95^{\circ} \mathrm{C}$; $30 \mathrm{~s}$ at $58{ }^{\circ} \mathrm{C}$; and finally, $1 \mathrm{~s}$ at $72{ }^{\circ} \mathrm{C}$ with acquisition mode (parameters of wavelength excitation and detection equal 465 and $510 \mathrm{~nm}$, respectively). The reaction for each gene was standardized against a standard curve, to estimate amplification efficiency. Standardization procedure included preparation of 10 -fold serial dilutions with controlled relative amount of targeted template. The efficiency of amplification was assessed based on a slope of the standard curve. Standard dilutions were amplified in separate wells, but within the same run. Then, the samples were subjected to qPCR with measurement of $C_{\mathrm{t}}$, and amplification efficiencies were automatically calculated and displayed on the analysis window of LightCycler 480 software, version 1.5.1.62 (Roche). The same software was also used for sample setup, real-time PCR analysis, and calculation of relative $C_{\mathrm{t}}$ values referred to as "ratios."

\section{Statistical methods}

The results are presented as medians, interquartile ranges, and non-outlier ranges. Normal distribution of the study variables was verified with Kolmogorov-Smirnov test with Lilliefors correction and based on visual inspection of plotted histograms. Variables with non-normal distributions (5-hmdC, 5-fdC, 5-cadC, 5-hmdU, 8-oxodG concentration and TETs, $A I D$ mRNA expression) were subjected to Box-Cox transformation prior to statistical analyses with parametric tests. Normalized data were subjected to one-way analysis of variance (ANOVA) followed by LSD and Tukey post hoc tests. Associations between pairs of variables were assessed based on Pearson correlation coefficients for raw or normalized data, where applicable. All statistical transformations and analyses were carried out with STATISTICA 13.1 PL [Dell Inc. (2016). Dell Statistica (data analysis software system), version 13. 
software.dell.com.]. The results were considered statistically significant at $P$ values lower than 0.05 .

\section{Results}

Levels of epigenetic modifications and 8-oxodGuo in DNA from tissue specimens

The highest levels of 5-mdC and 5-hmdC were found in normal colonic tissue, followed by IBD, AD, and CRC specimens (Fig. 1a, b); the level of 5-mdC in AD turned out to be significantly lower than in other tissues. In turn, CRC specimens were characterized by significantly lower levels of 5 - $\mathrm{fdC}$ than other samples (Fig. 1c). The level of 5-cadC in AD was significantly (2- to 2.5-fold) lower than in other tissues; in turn, the highest level of this modification was found in normal colonic tissue (Fig. 1d). We also analyzed possible association between level of the epigenetic modifications and tumor progression reflected in tumor stage from A to D. Significant decrease of 5-mdC, 5-hmdC, and 5-fdC was characteristic for early stage of CRC development (stage A), and no further changes were observed along the disease progression.

The highest levels of 5-hmdU and 8-oxodG were observed in IBD and AD and the lowest in CRC and normal colonic tissue; also, these intergroup differences were statistically significant (Fig. 1e, f).

Furthermore, significant correlations were found in the levels of 5-mdC, 5-cadC, 8-oxodG, and 5-hmdU between CRC and normal colon (Fig. 2).

We have analyzed relationship/correlation between age and 5-hmCyt (and other modifications). However in
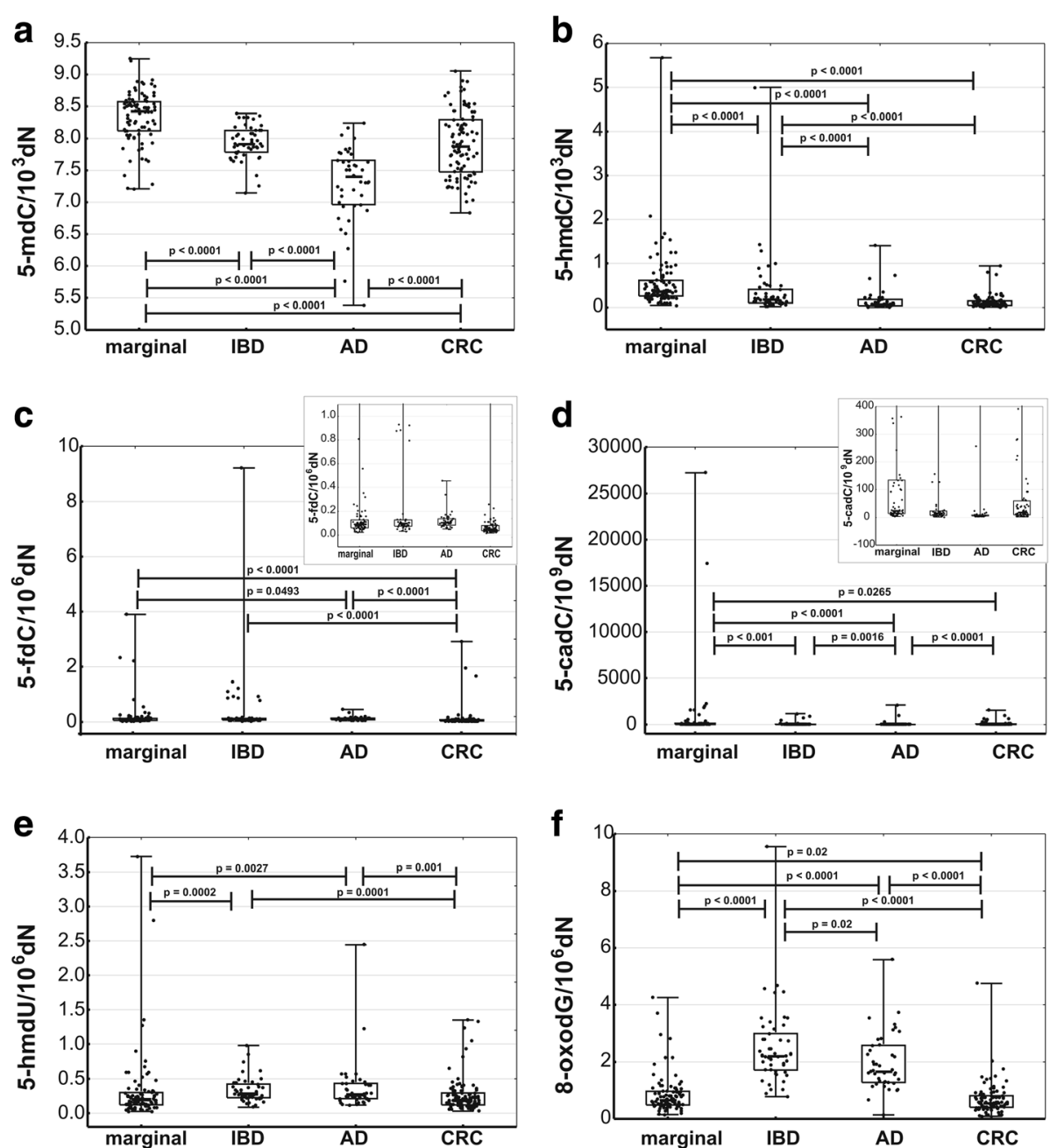

Fig. 1 Levels of DNA epigenetic modifications-5-mdC (a), 8-oxodG (b), 5-hmdC (c), 5-fdC (d), 5-cadC (e), and 5-hmdU (f) in normal colonic tissue ( $n=90)$; inflammatory lesions, IBD ( $n=49)$; polyps, AD $(n=39)$; and cancer tissue, CRC $(n=97)$. Marker in the center of the box represents median value. The length of each box (IQR, interquartile range) represents the range of values for $50 \%$ of the most typical observations, and its edges correspond to the first and third quartile. Whiskers represent variance outside the upper and lower quartile. $P$ value was determined with Mann-Whitney $U$ test 

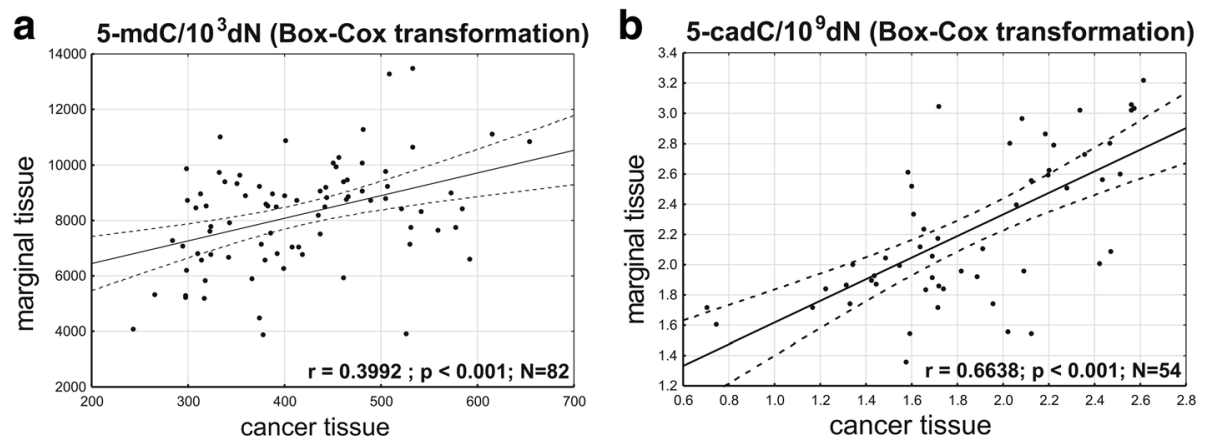

C $8-0 x o d G / 10^{6} \mathrm{dN}$ (Box-Cox transformation)
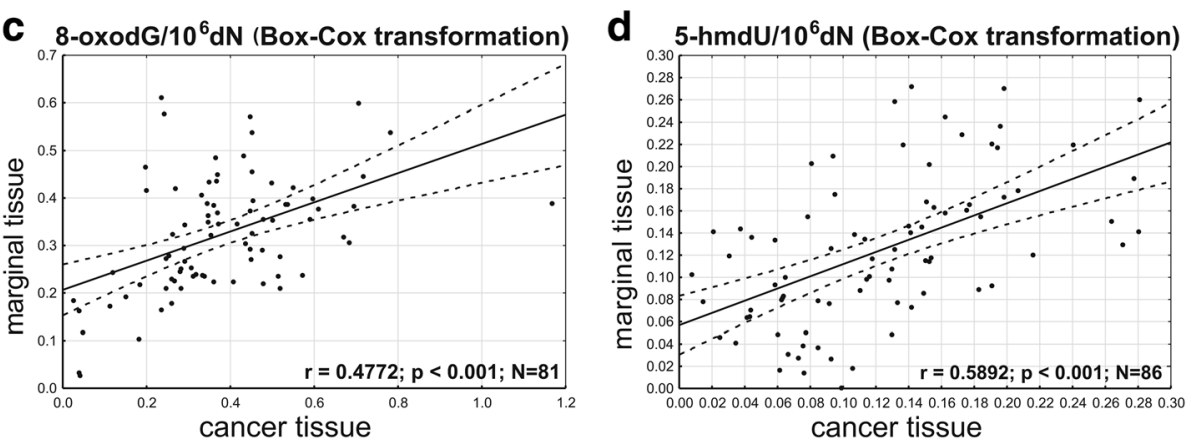

Fig. 2 Correlations between the levels of DNA epigenetic modifications, 5-mdC (a), 5-cadC (b), 8-oxodG (c), and 5-hmdU (d), in normal colonic tissue and cancer tissue

CRC patients as well as in other groups, no such correlation was found.

\section{Expression of TET and AID mRNA}

The expression of TET1 in AD and CRC was significantly weaker than in normal colonic tissue and IBD (Fig. 3a). The expression of TET2 in normal colonic tissue was significantly weaker than in $\mathrm{AD}$ and $\mathrm{CRC}$; moreover, a significant difference was found in TET2 expressions in IBD and AD (Fig. 3b). The examined tissues did not differ significantly in terms of TET3 expressions (Fig. 3c). Irrespective of the examined tissue, the levels of AID mRNA were very low or below the detection threshold. Nevertheless, the levels of AID mRNA in CRC turned out to be significantly lower than in other tissues (Fig. 3d). No statistically significant correlations were found between TETs expression and epigenetic DNA modifications (data not shown).

\section{Immunohistochemical analysis of protein expression}

Immunoreactivity to anti-TET1 antibodies in CRC turned out to be significantly lower than in normal colonic tissue (Fig. 4). In the case of TET2, immunohistochemical analysis was on the borderline of statistical significance with $p=0.06$.

\section{Discussion}

Although a molecular link between adenomas, chronic inflammation, and carcinogenesis is still not completely understood, a major contributor to CRC development seems to be aberrant methylation of DNA and oxidatively damaged DNA. A growing body of evidence suggests that decreased levels of 5-mdC (for review, see: [21]) and 5-hmdC [22] may be found not only in human malignancies but also in their precursor lesions, such as adenomas. This implies that the level of this modification may decrease gradually throughout carcinogenesis. However, it is still unclear if hypomethylation is a late or early event in cancer development, and whether this process is directly involved in carcinogenesis (for review, see: [21]). Therefore, the aim of this study was to verify if $\mathrm{CRC}$ and its precursor lesions differ from normal colonic tissue in the levels of DNA epigenetic modifications.

Similar to previous studies, we demonstrated that 5-hmdC level in CRC was several times lower than in normal colonic tissue. However, the level of this modification in cancer precursor lesions still raises some controversies. In some studies, the levels of 5 -hmdC in benign lesions were shown to be lower than in normal tissues [7, 22]. However, in a recent study, the results of which were published in Cell [6], melanocytes forming benign nevi showed relatively high levels of 5-hmdC, whereas a significant decrease or complete loss of this epigenetic mark was observed in melanoma cells. It should be stressed that in all these studies, 5 -hmdC was determined with a less accurate semiquantitative 

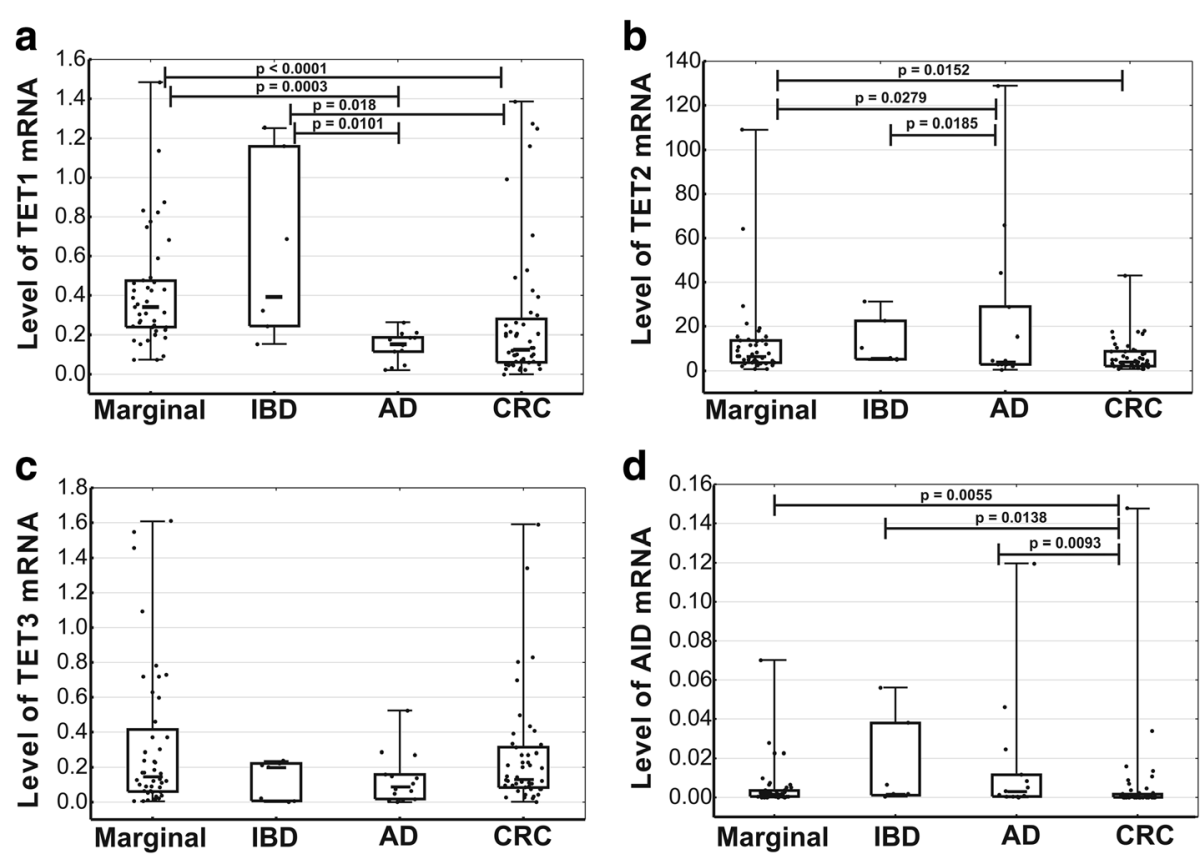

Fig. 3 Expressions of TET1 (a), TET2 (b), TET3 (c), and AID (d) mRNA in normal colonic tissue, cancer tissue (CRC, $n=49)$, inflammatory lesions (IBD, $n=7)$, and polyps ( $A D, n=14)$. Marker in the center of the box represents median value. The length of each box (IQR, interquartile range) represents the range of values for $50 \%$ of the most typical observations, and its edges correspond to the first and third quartile. Whiskers represent variance outside the upper and lower quartile. $P$ value was determined with Mann-Whitney $U$ test

method. Our present study, involving highly accurate quantitative technique for 5 -hmdC determination, demonstrated that the level of this modification in AD and CRC was essentially the same, approximately four times lower than in normal colonic tissue. These findings are consistent with the results published by Uribe-Lewis et al. [22], who also showed that 5-hmdC levels in CRC and adenoma were substantially lower than in normal colon. However, to the best of our knowledge, our present study was the first one to show that the level of 5-hmdC in IBD was significantly lower than in normal colonic tissue, at a midrange between the values found in this material and in CRC (Fig. 1b).

Interestingly, a significant decrease in 5 -fdC content was observed solely in CRC, and the level of this modification in both types of precursor lesions, IBD and AD, was essentially the same as in normal colonic tissue (Fig. 1c). Furthermore, both IBD and AD were characterized by significantly higher levels of 8-oxodG (the marker of oxidative stress) than CRC and normal colon (Fig. 1f). It should be remembered that the induction of oxidative stress in a cell culture was previously shown to contribute to a decrease in 5-hmdC level [23].

The level of another higher-order oxidative modification of 5-mdC, i.e., 5-cadC, was significantly higher in CRC than in AD or IBD (Fig. 1a, d). Furthermore, we found a significant correlation between the levels of this modification in CRC and normal colonic tissue (Fig. 2).
Previous semiquantitative studies with specific antibodies demonstrated elevated levels of 5-cadC in human breast cancer and gliomas [24].

Recent evidence suggests that oncogenic transcription factors, Myc and Max, and perhaps also an array of regulatory proteins, can specifically recognize 5-cadC, having lesser affinity for 5-fdC and showing only a trace of affinity towards 5-mdC and 5-hmdC. It should be remembered that dysregulation of MYC-MAX transcriptional network is a common mechanism driving progression of human malignancies [25]. This may at least partially explain higher levels of 5-cadC found in cancer tissue. Moreover, Xiong et al. [26] showed recently that Sall4, an oncogenic protein being overexpressed in colon cancer [27], may cooperate with TET2, catalyzing oxidation of 5-hmdC and contributing to formation of 5-cadC. Another study demonstrated that TET3 may specifically bind to 5 -cadC, initiating BER pathway and thus activating the process of demethylation [28].

Intriguingly, the analyses of associations between overall survival and the levels of epigenetic modifications in CRC patients demonstrated that the only correlation of longer survival was low level of 5-cadC in marginal tissue (Fig. 5). Hence, an important question arises why this parameter is a meaningful predictor of longer survival in cancer patients? Although histopathological examination did not demonstrate presence of cancer cells in marginal/normal tissue, the molecular assays 


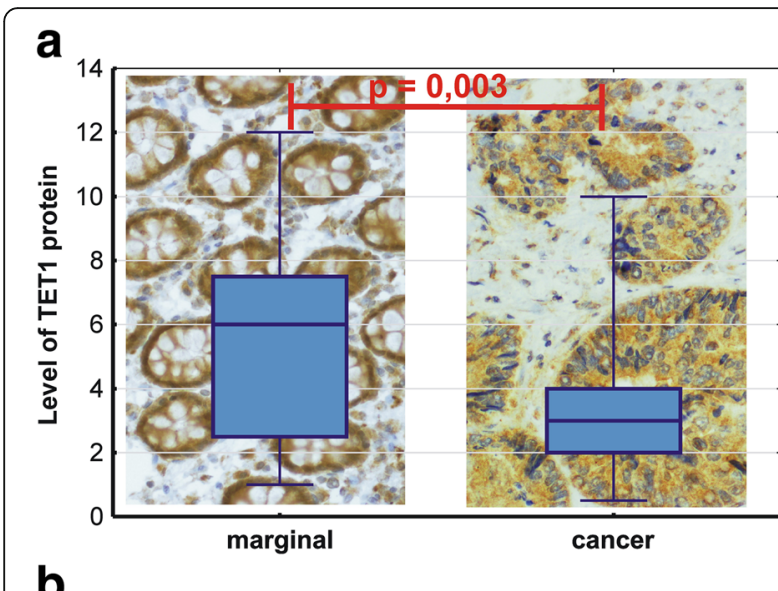

b
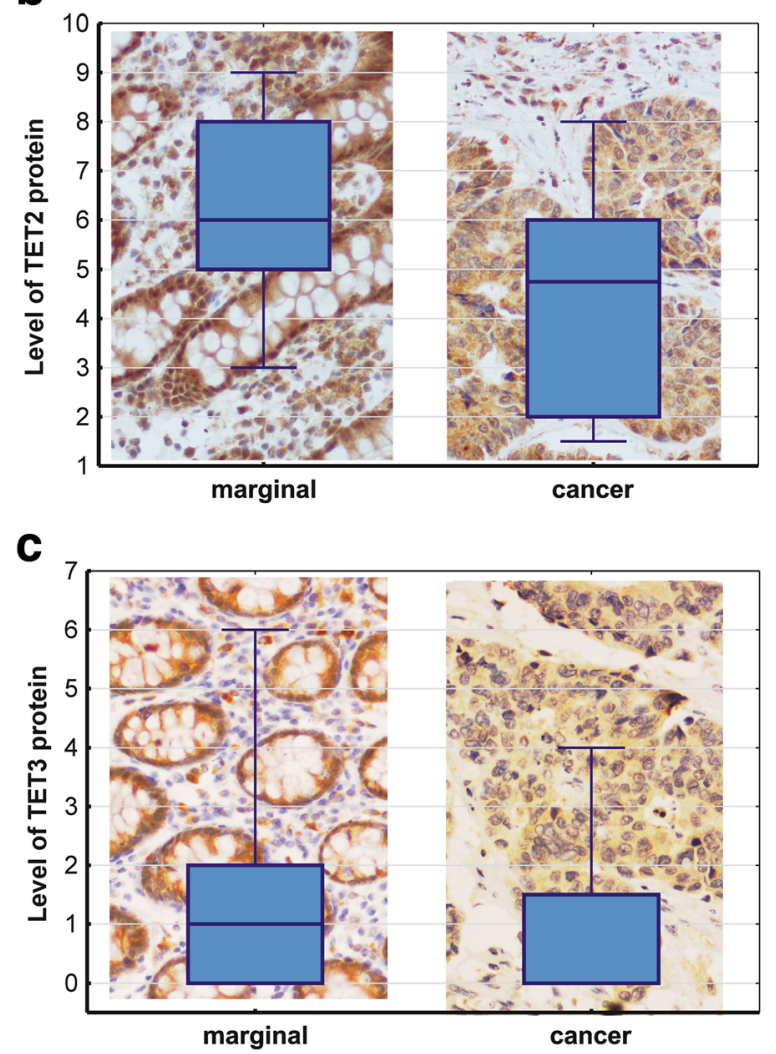

Fig. 4 Expressions of TET1 (a), TET2 (b), and TET3 (c) protein in normal colonic tissue and cancer tissue (CRC, $n=19)$. Marker in the center of the box represents median value. The length of each box (IQR, interquartile range) represents the range of values for $50 \%$ of the most typical observations, and its edges correspond to the first and third quartile. Whiskers represent variance outside the upper and lower quartile. $P$ value was determined with Mann-Whitney $U$ test

detected the cells being clonally related to the tumor (field cancerization) [29]. Moreover, in the case of CRC colon marginal tissue, field cancerization may involve up to $10-\mathrm{cm}$ patches [30]. Therefore, the relatively large specimen of marginal tissue used for DNA isolation likely corresponded to the area of field cancerization. Considering a strong correlation between 5-cadC levels in CRC and normal colonic tissue and a relatively high content of this modification in CRC, one can expect that marginal tissue with lower level of 5-cadC is less likely to contain cancer cells.

Both CRC and its precursor lesions, especially $A D$, showed significantly lower levels of 5-mdC than normal colonic tissue (Fig. 1). A dramatic decrease in 5-mdC and 5-hmdC levels in AD may contribute to genomic instability and thus represent a decisive step in CRC development. Interestingly, a substantial decrease in the levels of these modifications in $\mathrm{AD}$ (observed also in IBD specimens) co-existed with an increase in 5-hmU, 8-oxodG, and 5 -fdC content. This suggests that the decrease in 5-mdC level observed in cancer precursor lesions may be associated with the recently proposed phenomenon of processive DNA demethylation. Plausibly, 5-fdC, 5-hmd, and perhaps also 8-oxodG initiate processive demethylation of DNA, as proposed by Franchini et al. [31, 32]. In line with this hypothesis, an alternative pathway, the so-called processive DNA demethylation, exists aside from the active process involved in local and specific demethylation of DNA. According to the authors of this hypothesis, a single initiating event (such as a certain mismatch, e.g., 5-hmUra-G) may trigger processive demethylation of numerous 5-mdCs (and perhaps also 5-hmdCs) on the same locus via long-path BER, DNA mismatch repair (MMR), or nucleotide excision repair (NER) pathway. Recent experiments with cell-free extracts and circular heteroduplex DNA substrate demonstrated that $5-\mathrm{hmU}$ may trigger the removal of distant epigenetic modifications (5-mdC and 5-hmdC) on MMR- and long-path BER-dependent pathway [33].

Our present study showed that the expression of TET1 mRNA in CRC and AD was significantly weaker than in IBD and normal colon (Fig. 3). Furthermore, CRC and AD showed significantly lower levels of TET2 and AID mRNA than normal colonic tissue. However, at a protein level, the only significant difference between the examined tissues was found in the case of TET1, significantly more abundant in normal colon than in CRC.

A main factor contributing to a decrease in the activity of TET proteins are mutations in catalytic domains of these enzymes [34]. Another reason behind the reduced activity of TETs may be an inhibitory effect of accumulated onco-metabolites, such as 2-OH-glutarate [35, 36], resulting primarily from the presence of IDH1/2 mutations. However, these mutations were observed mainly in hematopoietic malignances and are rare or completely absent in solid tumors, such as CRC [37-39]. This implies that a decrease in 5 -hmdC level may be caused by other factors than TET/IDH mutations, for example oxidative stress. Indeed, recent evidence suggests that oxidative stress may contribute to post-translational 


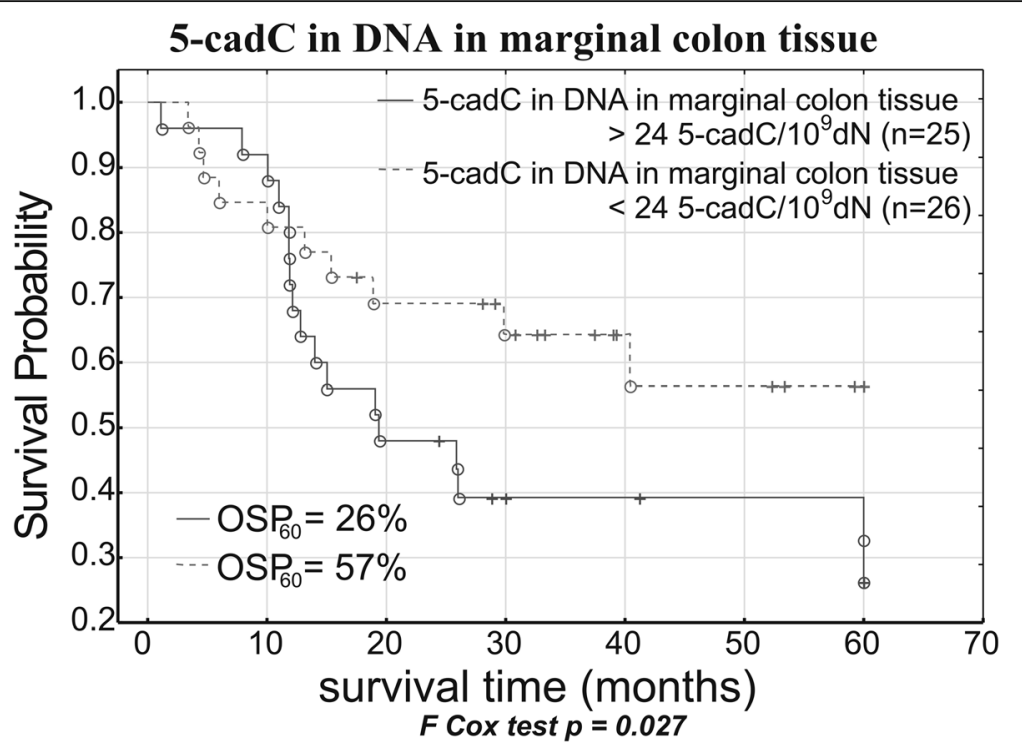

Fig. 5 Relation between the level of 5-cadC in marginal colon tissue and survival of CRC patients following surgery

modulation of TET2 [40]. Recently, it was demonstrated that the inhibition of TET proteins may be a direct consequence of hypoxia [41]. Hypoxia is a common phenomenon in solid tumors, which may at least partially explain a decrease in 5 -hmdC and 5-fdC levels observed in CRC. Interestingly, hypoxia increases overall oxidative stress and can change redox status of the cell [42].

Since the shape of TET co-substrates (2-ketoglutarate, $\mathrm{Fe}^{+2}$ ) depends on the redox state of the cell, the change in the activity of these enzymes may reflect the severity of oxidative stress. Furthermore, it cannot be excluded that also superoxide $\left(\mathrm{O}^{-2}\right)$, an anion radical of dioxygen and the precursor of free radicals, plays an important role in TET-mediated active DNA demethylation [43, 44]. Thus, changes in the activity of TET proteins may result from a persistent increase in the severity of oxidative stress, which promotes aberrant generation of DNA epigenetic modifications during iterative oxidation of 5-mdC. In this study, we found elevated levels of 8-oxodG in IBD and AD. Of note, level of 8-oxodG in DNA may directly inform about oxidative stress in nuclei of cells, where epigenetic processes take place. Recently, it was also demonstrated that 8-oxodG may serve as a demethylation signal [45]: binding to 8-oxodG; OGG1 glycosylase may recruit TET1 which in turn may be involved in specific DNA demethylation in response to oxidative stress/oxidatively damaged DNA. This may at least partially explain a decrease in 5 -mdC level observed in $\mathrm{AD}$ and IBD, i.e., in precursor lesions characterized by elevated levels of 8-oxodG.

\section{Conclusion}

Our hereby presented findings suggest that a complex relationship between aberrant pattern of DNA epigenetic modification and cancer development does not depend solely on the transcriptional status of TET proteins, but also on the characteristics of premalignant/malignant cells. This in turn implies that epigenetic modification of DNA is linked to oxidative stress. However, the exact character of this complex relationship is still poorly understood.

Our findings are consistent with the results of previous studies, showing that aberrant methylation of DNA occurs at very early stages of CRC development. Moreover, the hereby presented data add to existing evidence, showing that a decrease in the level of epigenetic marks is characteristic for early stages of CRC development, and further progression of the tumor is not associated with any additional changes in these parameters. To the best of our knowledge, this study was the first one to show that CRC, AD, and IBD had their unique epigenetic marks, distinguishing them from each other as well as from normal colonic tissue: (i) IBD was characterized by the highest level of 8-oxodG among all analyzed tissues, as well as by a decrease in 5 -hmdC and 5 - $\mathrm{mdC}$ levels (at a midrange between normal colon and $\mathrm{CRC}$ ); (ii) $\mathrm{AD}$ had the lowest levels of 5-hmdC and 5-mdC of all examined tissues and showed an increase in 8-oxodG and 5-hmdU levels; (iii) CRC was characterized by lower levels of 5 -hmdC and 5-mdC, the lowest level of 5-fdC among all analyzed tissues, and relatively high content of 5-cadC. This implies that a decrease in 5-hmdC level is not a unique feature of CRC (as previously reported) 
and can be also found in its precursor lesions, in particular in $\mathrm{AD}$. The mechanism behind a substantial decrease in 5 - $\mathrm{fdC}$ generation at advanced stages of carcinogenesis is still unclear, and the same refers to the consequences of this phenomenon. A recent observation that 5 - $\mathrm{fdC}$ is rich in active enhancers involved in tissue development/differentiation [46] sheds a new light on these relationships, implying that reduced level of this modification may be a characteristic feature of largely undifferentiated cancer cells.

It cannot be excluded that the analysis of a larger spectrum of DNA epigenetic modifications, rather than solely 5 -hmdC, supported by a transcriptional information, might provide a better insight in carcinogenesis, risk factors of CRC, and perhaps also therapy of this malignancy.

\section{Additional files}

Additional file 1: Table S1. Characteristics of antibodies used in immunohistochemical staining. (PDF $499 \mathrm{~kb}$ )

Additional file 2: Table S2. Transition patterns specific detector settings and sources of standards for analyzed compounds (relative response ratio = area under the peak of qualifierion/area under the peak of quantifier ion). (PDF $82 \mathrm{~kb}$ )

Additional file 3: Table S3. Primers and short hydrolysis probes used for TETs and AID mRNA expression analysis. (PDF $442 \mathrm{~kb}$ )

\section{Abbreviations}

2D-UPLC-MS/MS: Two-dimensional ultra-performance liquid chromatography with tandem mass spectrometry; 5-cadC: 5-Carboxy-2'deoxycytidine; 5-fdC: 5Formyl-2'-deoxycytidine; 5-hmdC: 5-Hydroxymethyl-2'-deoxycytidine; 5hmdU: 5-(Hydroxymethyl)-2'-deoxyuridine; 5-mdC: 5-Methyl-2'-deoxycytidine; 8-oxodG: 8-Oxo-2'-deoxyguanosine; AD: Adenoma; AID: Activation-induced cytidine deaminase protein; CRC: Colorectal cancer; FFPE: Formaldehydefixed paraffin-embedded; IBD: Inflammatory bowel disease; TET: Ten-eleven translocation protein; TMA: Tissue microarrays

\section{Funding}

This work was supported by the Polish National Science Center [grant no. 2013/09/B/NZ5/00767].

\section{Availability of data and materials}

The datasets used and/or analyzed during the current study are available from the corresponding author on reasonable request.

\section{Authors' contributions}

$\mathrm{RO}, \mathrm{AM}, \mathrm{MF}, \mathrm{ZB}, \mathrm{MK}, \mathrm{DG}$, and TD designed the research. ZB, MK, and ALi recruited the participants of the study. $A M$ and $M B$ performed the immunochemical analysis. TD, MS, EZ, MM, AS, JS, MG, KL, and ALa performed the isolation of DNA and its hydrolysis to deoxynucleosides. DG and MS performed determination of the epigenetic modifications in DNA. TD, JG, and KL performed isolation of RNA and gene expression analysis. TD, DG, and MF performed statistical analysis. TD, $M F$, and $R O$ supervised the research. TD and RO wrote the manuscript. RO, AM, $M F, Z B, M K, D G$, and TD revised and edited the manuscript. All authors read and approved the final manuscript.

\section{Ethics approval and consent to participate}

The study was conducted in accordance with the Declaration of Helsinki, its protocol was approved by the Local Bioethics Committee at Collegium Medicum, Nicolaus Copernicus University in Bydgoszcz (Poland), and written informed consent was sought from all the subjects.

\section{Competing interests}

The authors declare that they have no competing interests.

\section{Publisher's Note}

Springer Nature remains neutral with regard to jurisdictional claims in published maps and institutional affiliations.

\section{Author details}

${ }^{1}$ Department of Clinical Biochemistry, Faculty of Pharmacy, Collegium Medicum in Bydgoszcz, Nicolaus Copernicus University in Torun, Torun, Poland. ${ }^{2}$ Department of Clinical Pathomorphology, Faculty of Medicine, Collegium Medicum in Bydgoszcz, Nicolaus Copernicus University in Torun, Torun, Poland. ${ }^{3}$ Department of Surgery, Faculty of Medicine, Collegium Medicum in Bydgoszcz, Nicolaus Copernicus University in Torun, Torun, Poland. ${ }^{4}$ Department of Vascular Diseases and Internal Medicine, Faculty of Health Sciences, Collegium Medicum in Bydgoszcz, Nicolaus Copernicus University in Torun, Torun, Poland. ${ }^{5}$ Department of Oncologic Pathology and Prophylaxis, Poznan University of Medical Sciences and Greater Poland Cancer Center, Poznan, Poland. ${ }^{6}$ Department of Otolaryngology and Laryngeal Oncology, K. Marcinkowski University of Medical Sciences, Poznan, Poland. 'Department of Clinical Biochemistry, Collegium Medicum in Bydgoszcz, Nicolaus Copernicus University, Karlowicza 24, 85-095 Bydgoszcz, Poland.

Received: 4 December 2017 Accepted: 17 May 2018

Published online: 30 May 2018

\section{References}

1. Feng $\mathrm{S}$, Jacobsen $\mathrm{SE}$, Reik W. Epigenetic reprogramming in plant and animal development. Science. 2010;330:622-7.

2. Bhutani N, Burns DM, Blau HM. DNA demethylation dynamics. Cell. 2011; 146:866-72.

3. Tahiliani M, Koh KP, Shen Y, Pastor WA, Bandukwala H, Brudno Y, Agarwal S, lyer LM, Liu DR, Aravind L, Rao A. Conversion of 5-methylcytosine to 5hydroxymethylcytosine in mammalian DNA by MLL partner TET1. Science. 2009;324:930-5.

4. Pfaffeneder T, Spada F, Wagner M, Brandmayr C, Laube SK, Eisen D, Truss M, Steinbacher J, Hackner B, Kotljarova O, Schuermann D, Michalakis S, Kosmatchev O, Schiesser S, Steigenberger B, Raddaoui N, Kashiwazaki G, Muller U, Spruijt CG, Vermeulen M, Leonhardt H, Schar P, Muller M, Carell T. Tet oxidizes thymine to 5-hydroxymethyluracil in mouse embryonic stem cell DNA. NatChemBiol. 2014;10:574-81.

5. Jin SG, Jiang Y, Qiu R, Rauch TA, Wang Y, Schackert G, Krex D, Lu Q, Pfeifer GP. 5-Hydroxymethylcytosine is strongly depleted in human cancers but its levels do not correlate with IDH1 mutations. Cancer Res. 2011;71:7360-5.

6. Lian CG, Xu Y, Ceol C, Wu F, Larson A, Dresser K, Xu W, Tan L, Hu Y, Zhan Q Lee CW, Hu D, Lian BQ, Kleffel S, Yang Y, Neiswender J, Khorasani AJ, Fang R, Lezcano C, Duncan LM, Scolyer RA, Thompson JF, Kakavand H, Houvras $Y$, Zon LI, Mihm MC Jr, Kaiser UB, Schatton T, Woda BA, Murphy GF, Shi YG. Loss of 5-hydroxymethylcytosine is an epigenetic hallmark of melanoma. Cell. 2012;150:1135-46.

7. Yang H, Liu Y, Bai F, Zhang JY, Ma SH, Liu J, Xu ZD, Zhu HG, Ling ZQ, Ye D, Guan KL, Xiong Y. Tumor development is associated with decrease of TET gene expression and 5-methylcytosine hydroxylation. Oncogene. 2013;32: 663-9.

8. Chen ML, Shen F, Huang W, Qi JH, Wang Y, Feng YQ, Liu SM, Yuan BF. Quantification of 5-methylcytosine and 5-hydroxymethylcytosine in genomic DNA from hepatocellular carcinoma tissues by capillary hydrophilic-interaction liquid chromatography/quadrupole TOF mass spectrometry. Clin Chem. 2013;59:824-32.

9. Cimmino L, Abdel-Wahab O, Levine RL, Aifantis I. TET family proteins and their role in stem cell differentiation and transformation. Cell Stem Cell. 2011;9:193-204.

10. Kraus S, Arber N. Inflammation and colorectal cancer. CurrOpinPharmacol. 2009;9:405-10.

11. Iurlaro M, Ficz G, Oxley D, Raiber EA, Bachman M, Booth MJ, Andrews S, Balasubramanian S, Reik W. A screen for hydroxymethylcytosine and formylcytosine binding proteins suggests functions in transcription and chromatin regulation. Genome Biol. 2013;14:R119. 
12. Spruijt CG, Gnerlich F, Smits AH, Pfaffeneder $T$, Jansen PW, Bauer C, Munzel M, Wagner M, Muller M, Khan F, Eberl HC, Mensinga A, Brinkman AB, Lephikov K, Muller U, Walter J, Boelens R, van $\|_{\text {, }}$ Leonhardt H, Carell T, Vermeulen M. Dynamic readers for 5(hydroxy)methylcytosine and its oxidized derivatives. Cell. 2013;152: $1146-59$.

13. Gackowski D, Starczak M, Zarakowska E, Modrzejewska M, Szpila A, Banaszkiewicz Z, Olinski R. Accurate, direct, and high-throughput analyses of a broad spectrum of endogenously generated DNA base modifications with isotope-dilution two-dimensional ultraperformance liquid chromatography with tandem mass spectrometry: possible clinical implication. AnalChem. 2016;88:12128-36.

14. Bodnar M, Szylberg L, Kazmierczak W, Marszalek A. Tumor progression driven by pathways activating matrix metalloproteinases and their inhibitors. JOral PatholMed. 2015:44:437-43.

15. Bodnar M, Luczak M, Bednarek K, Szylberg L, Marszalek A, Grenman R, Szyfter K, Jarmuz-Szymczak M, Giefing M. Proteomic profiling identifies the inorganic pyrophosphatase (PPA1) protein as a potential biomarker of metastasis in laryngeal squamous cell carcinoma. AminoAcids. 2016;48: 1469-76.

16. Bodnar M, Burduk $P$, Antosik $P$, Jarmuz-Szymczak M, Wierzbicka M, Marszalek A. Assessment of BRAF V600E (VE1) protein expression and BRAF gene mutation status in codon 600 in benign and malignant salivary gland neoplasms. JOral PatholMed. 2017:46:340-5.

17. Uhlen M, Oksvold P, Fagerberg L, Lundberg $E$, Jonasson $K$, Forsberg $M$, Zwahlen M, Kampf C, Wester K, Hober S, Wernerus H, Bjorling L, Ponten F. Towards a knowledge-based human protein atlas. NatBiotechnol. 2010;28: 1248-50.

18. Remmele W, Stegner HE. Recommendation for uniform definition of an immunoreactive score (IRS) for immunohistochemical estrogen receptor detection (ER-ICA) in breast cancer tissue. Pathologe. 1987;8:138-40.

19. Burduk PK, Bodnar M, Sawicki P, Szylberg L, Wisniewska E, Kazmierczak W, Martynska M, Marszalek A. Expression of metalloproteinases 2 and 9 and tissue inhibitors 1 and 2 as predictors of lymph node metastases in oropharyngeal squamous cell carcinoma. Head Neck. 2015;37:418-22.

20. Guz J, Foksinski M, Siomek A, Gackowski D, Rozalski R, Dziaman T, Szpila A, Olinski R. The relationship between 8-oxo-7,8-dihydro-2'-deoxyguanosine level and extent of cytosine methylation in leukocytes DNA of healthy subjects and in patients with colon adenomas and carcinomas. MutatRes. 2008:640:170-3.

21. Herceg Z, Vaissiere T. Epigenetic mechanisms and cancer: an interface between the environment and the genome. Epigenetics. 2011;6:804-19.

22. Uribe-Lewis S, Stark R, Carroll T, Dunning MJ, Bachman M, Ito Y, Stojic L, Halim S, Vowler SL, Lynch AG, Delatte B, de Bony EJ, Colin L, Defrance M, Krueger F, Silva AL, Ten HR, Ibrahim AE, Fuks F, Murrell A. 5hydroxymethylcytosine marks promoters in colon that resist DNA hypermethylation in cancer. Genome Biol. 2015;16:69.

23. Delatte B, Jeschke J, Defrance M, Bachman M, Creppe C, Calonne E, Bizet M, Deplus R, Marroqui L, Libin M, Ravichandran M, Mascart F, Eizirik DL, Murrell A, Jurkowski TP, Fuks F. Genome-wide hydroxymethylcytosine pattern changes in response to oxidative stress. SciRep. 2015;5:12714.

24. Eleftheriou M, Pascual AJ, Wheldon LM, Perry C, Abakir A, Arora A, Johnson AD, Auer DT, Ellis IO, Madhusudan S, Ruzov A. 5-Carboxylcytosine levels are elevated in human breast cancers and gliomas. ClinEpigenetics. 2015;7:88.

25. Wang D, Hashimoto $H$, Zhang X, Barwick BG, Lonial S, Boise LH, Vertino PM, Cheng X. MAX is an epigenetic sensor of 5-carboxylcytosine and is altered in multiple myeloma. Nucleic Acids Res. 2017;45:2396-407.

26. Xiong J, Zhang Z, Chen J, Huang H, Xu Y, Ding X, Zheng Y, Nishinakamura R, Xu GL, Wang H, Chen S, Gao S, Zhu B. Cooperative action between SALL4A and TET proteins in stepwise oxidation of 5-methylcytosine. Mol Cell. 2016;64:913-25.

27. Cheng J, Deng R, Zhang P, Wu C, Wu K, Shi L, Liu X, Bai J, Deng M, Shuai X, Gao J, Wang G, Tao K. miR-219-5p plays a tumor suppressive role in colon cancer by targeting oncogene Sall4. Oncol Rep. 2015;34:1923-32.

28. Jin SG, Zhang ZM, Dunwell TL, Harter MR, Wu X, Johnson J, Li Z, Liu J, Szabo PE, Lu Q, Xu GL, Song J, Pfeifer GP. Tet3 reads 5-carboxylcytosine through its CXXC domain and is a potential guardian against neurodegeneration. Cell Rep. 2016;14:493-505.

29. Braakhuis BJ, Tabor MP, Kummer JA, Leemans CR, Brakenhoff RH. A genetic explanation of Slaughter's concept of field cancerization: evidence and clinical implications. Cancer Res. 2003;63:1727-30.
30. Hawthorn L, Lan L, Mojica W. Evidence for field effect cancerization in colorectal cancer. Genomics. 2014;103:211-21.

31. Franchini DM, Chan CF, Morgan H, Incorvaia E, Rangam G, Dean W, Santos F, Reik W, Petersen-Mahrt SK. Processive DNA demethylation via DNA deaminase-induced lesion resolution. PLoSOne. 2014;9:e97754.

32. Olinski R, Starczak M, Gackowski D. Enigmatic 5-hydroxymethyluracil: oxidatively modified base, epigenetic mark or both? MutatResRevMutatRes. 2016;767:59-66

33. Grin I, Ishchenko AA. An interplay of the base excision repair and mismatch repair pathways in active DNA demethylation. Nucleic Acids Res. 2016;44: 3713-27.

34. Kohli RM, Zhang Y. TET enzymes, TDG and the dynamics of DNA demethylation. Nature. 2013;502:472-9.

35. Xu W, Yang H, Liu Y, Yang Y, Wang P, Kim SH, Ito S, Yang C, Wang P, Xiao MT, Liu LX, Jiang WQ, Liu J, Zhang JY, Wang B, Frye S, Zhang Y, Xu YH, Lei QY, Guan KL, Zhao SM, Xiong Y. Oncometabolite 2-hydroxyglutarate is a competitive inhibitor of alpha-ketoglutarate-dependent dioxygenases. Cancer Cell. 2011;19:17-30.

36. Figueroa ME, Bdel-Wahab O, Lu C, Ward PS, Patel J, Shih A, Li Y, Bhagwat N, Vasanthakumar A, Fernandez HF, Tallman MS, Sun Z, Wolniak K, Peeters JK, Liu W, Choe SE, Fantin VR, Paietta E, Lowenberg B, Licht JD, Godley LA, Delwel R, Valk PJ, Thompson CB, Levine RL, Melnick A. Leukemic IDH1 and IDH2 mutations result in a hypermethylation phenotype, disrupt TET2 function, and impair hematopoietic differentiation. Cancer Cell. 2010;18:55367.

37. Wu X, Zhang Y. TET-mediated active DNA demethylation: mechanism, function and beyond. NatRevGenet. 2017;18:517-34.

38. Kan Z, Jaiswal BS, Stinson J, Janakiraman V, Bhatt D, Stern HM, Yue P, Haverty PM, Bourgon R, Zheng J, Moorhead M, Chaudhuri S, Tomsho LP, Peters BA, Pujara K, Cordes S, Davis DP, Carlton VE, Yuan W, Li L, Wang W, Eigenbrot C, Kaminker JS, Eberhard DA, Waring P, Schuster SC, Modrusan Z, Zhang Z, Stokoe D, de Sauvage FJ, Faham M, Seshagiri S. Diverse somatic mutation patterns and pathway alterations in human cancers. Nature. 2010; 466:869-73

39. Seshagiri S, Stawiski EW, Durinck S, Modrusan Z, Storm EE, Conboy CB, Chaudhuri S, Guan Y, Janakiraman V, Jaiswal BS, Guillory J, Ha C, Dijkgraaf GJ, Stinson J, Gnad F, Huntley MA, Degenhardt JD, Haverty PM, Bourgon R, Wang W, Koeppen H, Gentleman R, Starr TK, Zhang Z, Largaespada DA, Wu TD, de Sauvage FJ. Recurrent R-spondin fusions in colon cancer. Nature. 2012:488:660-4.

40. Zhang YW, Wang Z, Xie W, Cai Y, Xia L, Easwaran H, Luo J, Yen RC, Li Y, Baylin SB. Acetylation enhances TET2 function in protecting against abnormal DNA methylation during oxidative stress. MolCell. 2017;65:323-35.

41. Thienpont B, Steinbacher J, Zhao H, D'Anna F, Kuchnio A, Ploumakis A, Ghesquiere B, Van DL, Boeckx B, Schoonjans L, Hermans E, Amant F, Kristensen VN, Koh KP, Mazzone M, Coleman ML, Carell T, Carmeliet P, Lambrechts $D$. Tumour hypoxia causes DNA hypermethylation by reducing TET activity. Nature. 2016;537:63-8.

42. Debevec T, Millet GP, Pialoux V. Hypoxia-induced oxidative stress modulation with physical activity. Front Physiol. 2017:8:84.

43. Afanas'ev I. Mechanisms of superoxide signaling in epigenetic processes: relation to aging and cancer. Aging Dis. 2015;6:216-27.

44. Cyr AR, Domann FE. The redox basis of epigenetic modifications: from mechanisms to functional consequences. AntioxidRedoxSignal. 2011;15:551-89.

45. Zhou X, Zhuang Z, Wang W, He L, Wu H, Cao Y, Pan F, Zhao J, Hu Z, Sekhar C, Guo Z. OGG1 is essential in oxidative stress induced DNA demethylation. Cell Signal. 2016;28:1163-71.

46. Iurlaro M, McInroy GR, Burgess HE, Dean W, Raiber EA, Bachman M, Beraldi D, Balasubramanian S, Reik W. In vivo genome-wide profiling reveals a tissue-specific role for 5-formylcytosine. Genome Biol. 2016;17:141. 